Acta Crystallographica Section E

Structure Reports

Online

ISSN 1600-5368

\section{N-Cyclohexyl-N-methylbenzene- sulfonamide}

\section{Zeeshan Haider, ${ }^{a}$ Islam Ullah Khan, ${ }^{\text {a* Muhammad }}$ Nadeem Arshad, ${ }^{a}$ Muhammad Shafiq ${ }^{a}$ and Caoyuan $\mathrm{Niu}^{\mathrm{b}}$ *}

aMaterials Chemistry Laboratory, Department of Chemistry, GC University, Lahore 54000, Pakistan, and ${ }^{\mathbf{b}}$ College of Sciences, Henan Agricultural University, Zhengzhou 450002, People's Republic of China

Correspondence e-mail: iukhan.gcu@gmail.com,niu_cy2000@yahoo.com.cn

Received 9 October 2009; accepted 13 October 2009

Key indicators: single-crystal X-ray study; $T=296 \mathrm{~K}$; mean $\sigma(\mathrm{C}-\mathrm{C})=0.003 \AA$; $R$ factor $=0.038 ; w R$ factor $=0.113 ;$ data-to-parameter ratio $=16.1$.

The title compound, $\mathrm{C}_{13} \mathrm{H}_{19} \mathrm{NO}_{2} \mathrm{~S}$, was synthesized by the reaction of $\mathrm{N}$-cyclohexylaminebenzenesulfonamide and methyl iodide. The crystal packing is stabilized by weak intermolecular $\mathrm{C}-\mathrm{H}$... O hydrogen bonds.

\section{Related literature}

Compounds containing cyclohexylamine have been reported to be activators of dopamine receptors in the central nervous system, see: Hacksell et al. (1981). For related structures, see: Arshad et al. (2008, 2009).

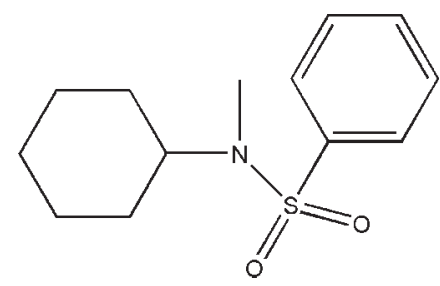

\section{Experimental}

Crystal data

$\mathrm{C}_{13} \mathrm{H}_{19} \mathrm{NO}_{2} \mathrm{~S}$

$M_{r}=253.35$
Monoclinic, $P 2_{1} / c$

$a=9.2729(5) \AA$

$b=12.1182(7) \AA$

$c=12.5801(7) \AA$

$\beta=109.103(2)^{\circ}$

$V=1335.79(13) \AA^{3}$

Data collection

Bruker APEXII CCD detector diffractometer

Absorption correction: multi-scan (SADABS; Bruker, 2005)

$T_{\min }=0.938, T_{\max }=0.979$

Refinement

$R\left[F^{2}>2 \sigma\left(F^{2}\right)\right]=0.038$

$w R\left(F^{2}\right)=0.113$

$S=1.08$

2489 reflections

$Z=4$

Mo $K \alpha$ radiation

$\mu=0.23 \mathrm{~mm}^{-1}$

$T=296 \mathrm{~K}$

$0.28 \times 0.12 \times 0.09 \mathrm{~mm}$

12741 measured reflections 2489 independent reflections 1864 reflections with $I>2 \sigma(I)$

$R_{\text {int }}=0.030$

Table 1

Hydrogen-bond geometry $\left(\AA,^{\circ}\right)$.

\begin{tabular}{lllll}
\hline$D-\mathrm{H} \cdots A$ & $D-\mathrm{H}$ & $\mathrm{H} \cdots A$ & $D \cdots A$ & $D-\mathrm{H} \cdots A$ \\
\hline $\mathrm{C} 2-\mathrm{H} 2 \cdots \mathrm{O} 2^{\mathrm{i}}$ & 0.93 & 2.52 & $3.268(3)$ & 137 \\
\hline
\end{tabular}

Symmetry code: (i) $x,-y+\frac{1}{2}, z+\frac{1}{2}$.

Data collection: APEX2 (Bruker, 2005); cell refinement: SAINT (Bruker, 2005); data reduction: $S A I N T$; program(s) used to solve structure: SHELXS97 (Sheldrick, 2008); program(s) used to refine structure: SHELXL97 (Sheldrick, 2008); molecular graphics: SHELXL97 and DIAMOND (Brandenburg, 2005); software used to prepare material for publication: SHELXL97.

Supplementary data and figures for this paper are available from the IUCr electronic archives (Reference: BT5092).

\section{References}

Arshad, M. N., Tahir, M. N., Khan, I. U., Ahmad, E. \& Shafiq, M. (2008). Acta Cryst. E64, o2380.

Arshad, M. N., Tahir, M. N., Khan, I. U., Shafiq, M. \& Ahmad, S. (2009). Acta Cryst. E65, o940.

Brandenburg, K. (2005). DIAMOND. Crystal Impact GbR. Bonn, Germany. Bruker (2005). APEX2, SAINT, and SADABS. Bruker AXS Inc., Madison, Wisconsin, USA.

Hacksell, U., Arvidsson, L.-E., Svensson, U., Nilsson, J. L. G., Sanchez, D., Wikstroem, H., Lindberg, P., Hjorth, S. \& Carlsson, A. (1981). J. Med. Chem. 24, 1475-1482.

Sheldrick, G. M. (2008). Acta Cryst. A64, 112-122. 


\section{supporting information}

Acta Cryst. (2009). E65, o2892 [https://doi.org/10.1107/S1600536809041762]

\section{$\mathrm{N}$-Cyclohexyl-N-methylbenzenesulfonamide}

\section{Zeeshan Haider, Islam Ullah Khan, Muhammad Nadeem Arshad, Muhammad Shafiq and Caoyuan Niu}

\section{S1. Comment}

Sulfonamide compounds have gained much importance due to their therapeutic applications. The compound containing cyclohexylamine has been reported to be an activator of dopamine receptors in the CNS (Hacksell et al., 1981). The title compound is a sulfonamide derivative of cyclohexylamine in continuation to our previous work (Arshad et al., 2008; Arshad et al., 2009).

The molecular structure of the title compound (I) is shown in Fig. 1. The mean plane of the benzene ring and that of the four essentially planar $\mathrm{C}$ atoms (C8, C9, C11, C12. Maximum deviation, $0.0132 \AA$ ) of the chair-form cyclohexyl ring have a dihedral angle of $24.26(9)^{\circ}$. Furthermore, there are intermolecular $\mathrm{C}-\mathrm{H} \cdots \mathrm{O}$ hydrogen bonds between the aromatic $\mathrm{H}$ atom $(\mathrm{H} 2)$ and one sulfonamide $\mathrm{O}$ atom $\left(\mathrm{O} 2^{\mathrm{i}}\right.$, symmetric code: see table 1) of neighboring molecules that contribute to the three-dimensional packing (Fig. 2).

\section{S2. Experimental}

Sodium hydride $(0.88 \mathrm{mmol})$ was taken in a round bottom flask and washed with $\mathrm{n}$-hexane so as to remove the mineral oil dispersant. A solution of $N$-cyclohexylamine benzene sulfonamide $(0.43 \mathrm{mmol})$ in $5 \mathrm{ml}$ of $\mathrm{N}, N$ dimethyl formamide was added. The mixture was stirred for half an hour at room temperature. Then, methyl iodide $(0.86 \mathrm{mmol})$ was added and stirring was continued for about $3 \mathrm{hrs}$ until the complete consumption of sulfonamide. The reaction was monitored by TLC. After the completion of the reaction the contents were transferred into the distilled water ice. The product precipitated and was separated by filtration and recrystallized from methanol. The melting point of the product was observed to be $353 \mathrm{~K}$ uncorrected.

\section{S3. Refinement}

All $\mathrm{H}$ atoms were placed in calculated positions and refined using a riding model $\left[\mathrm{C}-\mathrm{H}=0.93 \AA\right.$ and $U_{\text {iso }}(\mathrm{H})=1.2 U_{\text {eq }}(\mathrm{C})$ for aromatic $\mathrm{H}$ atoms; $\mathrm{C}-\mathrm{H}=0.98 \AA$ and $U_{\text {iso }}(\mathrm{H})=1.2 U_{\text {eq }}(\mathrm{C})$ for tertiary $\mathrm{CH} ; \mathrm{C}-\mathrm{H}=0.97 \AA$ and $U_{\text {iso }}(\mathrm{H})=1.2 U_{\text {eq }}(\mathrm{C})$ for $\mathrm{CH}_{2} ; \mathrm{C}-\mathrm{H}=0.96 \AA$ and $U_{\text {iso }}(\mathrm{H})=1.5 U_{\text {eq }}(\mathrm{C})$ for the methyl $\mathrm{H}$ atoms]. The final difference Fourier map had a highest peak at $0.71 \AA$ from atom $\mathrm{C} 1$ and a deepest hole at $0.71 \mathrm{~A} \AA$ from atom S1, but were otherwise featureless. 


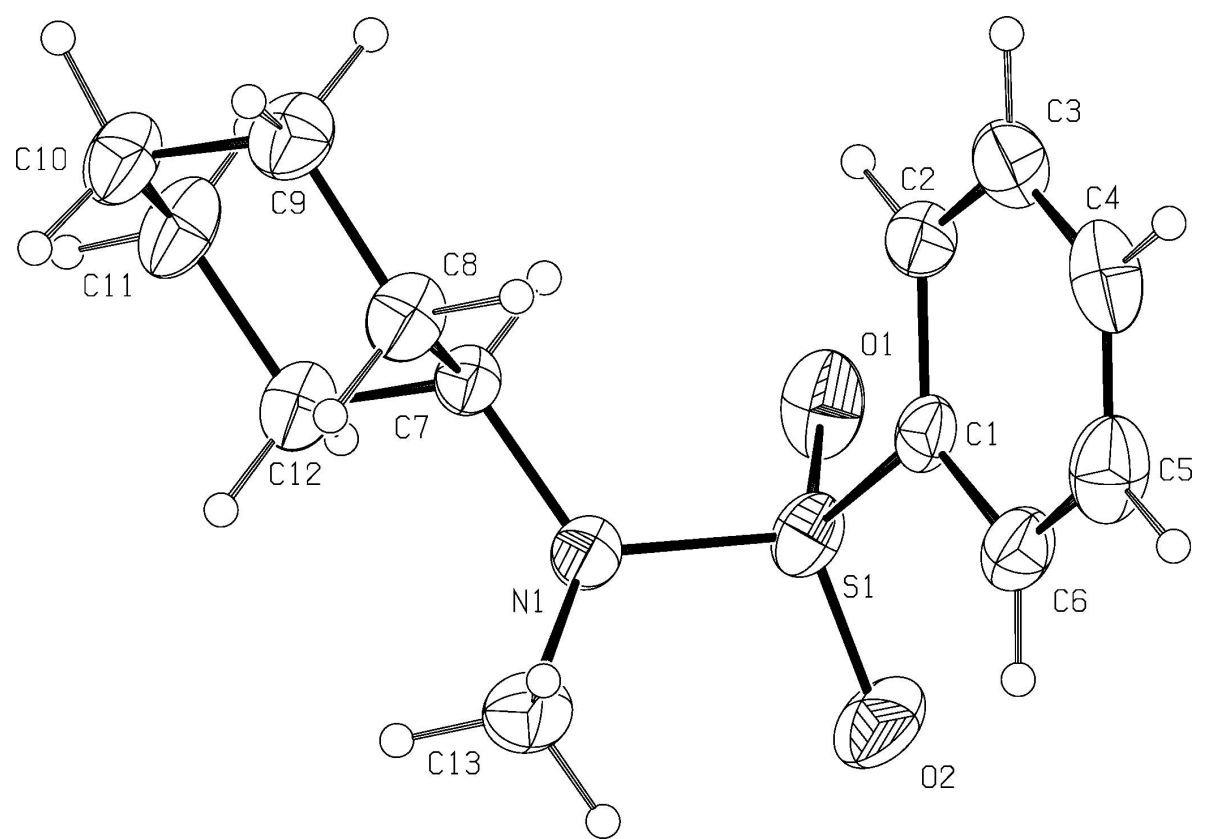

Figure 1

The molecular structure of the title compound showing the atom-labelling scheme. Displacement ellipsoids are drawn at the $30 \%$ probability level.

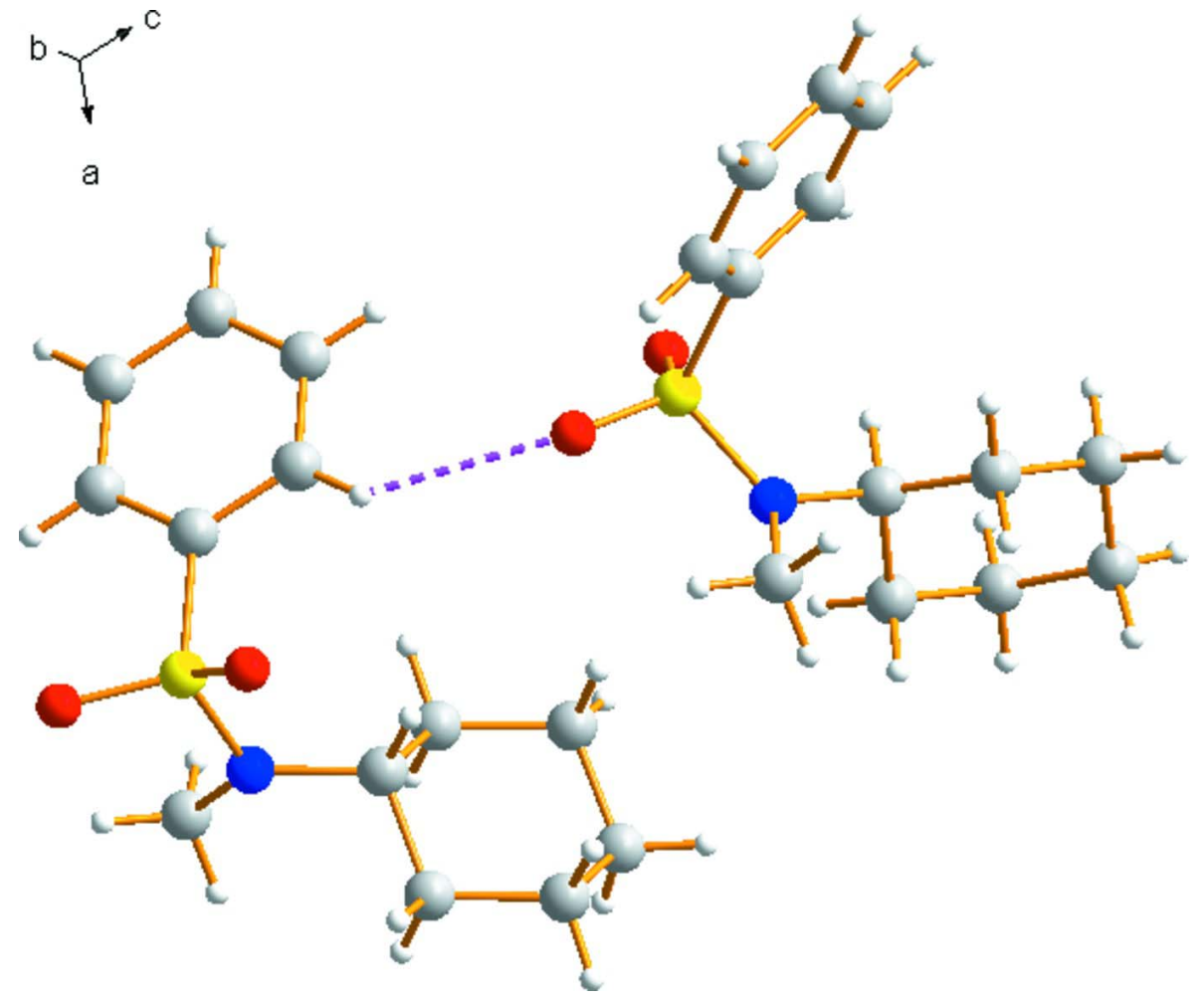

Figure 2

Diagram showing the intermolecular hydrogen bonds (indicated by pink dashed lines). 
$\mathrm{N}$-Cyclohexyl-N-methylbenzenesulfonamide

Crystal data

$\mathrm{C}_{13} \mathrm{H}_{19} \mathrm{NO}_{2} \mathrm{~S}$

$M_{r}=253.35$

Monoclinic, $P 2{ }_{1} / c$

Hall symbol: -P $2 \mathrm{ybc}$

$a=9.2729(5) \AA$

$b=12.1182(7) \AA$

$c=12.5801(7) \AA$

$\beta=109.103(2)^{\circ}$

$V=1335.79(13) \AA^{3}$

$Z=4$

\section{Data collection}

Bruker APEXII CCD detector diffractometer

Radiation source: fine-focus sealed tube Graphite monochromator

phi and $\omega$ scans

Absorption correction: multi-scan

(SADABS; Bruker, 2005)

$T_{\min }=0.938, T_{\max }=0.979$

\section{Refinement}

Refinement on $F^{2}$

Least-squares matrix: full

$R\left[F^{2}>2 \sigma\left(F^{2}\right)\right]=0.038$

$w R\left(F^{2}\right)=0.113$

$S=1.08$

2489 reflections

155 parameters

0 restraints

Primary atom site location: structure-invariant direct methods
$F(000)=544$

$D_{\mathrm{x}}=1.260 \mathrm{Mg} \mathrm{m}^{-3}$

Mo $K \alpha$ radiation, $\lambda=0.71073 \AA$

Cell parameters from 3617 reflections

$\theta=2.3-25.5^{\circ}$

$\mu=0.23 \mathrm{~mm}^{-1}$

$T=296 \mathrm{~K}$

Block, colourless

$0.28 \times 0.12 \times 0.09 \mathrm{~mm}$

12741 measured reflections

2489 independent reflections

1864 reflections with $I>2 \sigma(I)$

$R_{\text {int }}=0.030$

$\theta_{\text {max }}=25.5^{\circ}, \theta_{\min }=2.3^{\circ}$

$h=-10 \rightarrow 11$

$k=-14 \rightarrow 14$

$l=-13 \rightarrow 15$

Secondary atom site location: difference Fourier map

Hydrogen site location: inferred from

neighbouring sites

$\mathrm{H}$-atom parameters constrained

$w=1 /\left[\sigma^{2}\left(F_{\mathrm{o}}^{2}\right)+(0.0559 P)^{2}+0.2086 P\right]$

where $P=\left(F_{\mathrm{o}}^{2}+2 F_{\mathrm{c}}^{2}\right) / 3$

$(\Delta / \sigma)_{\max }<0.001$

$\Delta \rho_{\max }=0.16 \mathrm{e} \AA^{-3}$

$\Delta \rho_{\min }=-0.25$ e $\AA^{-3}$

Special details

Geometry. All e.s.d.'s (except the e.s.d. in the dihedral angle between two 1.s. planes) are estimated using the full covariance matrix. The cell e.s.d.'s are taken into account individually in the estimation of e.s.d.'s in distances, angles and torsion angles; correlations between e.s.d.'s in cell parameters are only used when they are defined by crystal symmetry. An approximate (isotropic) treatment of cell e.s.d.'s is used for estimating e.s.d.'s involving 1.s. planes.

Refinement. Refinement of $F^{2}$ against ALL reflections. The weighted $R$-factor $w R$ and goodness of fit $S$ are based on $F^{2}$, conventional $R$-factors $R$ are based on $F$, with $F$ set to zero for negative $F^{2}$. The threshold expression of $F^{2}>\sigma\left(F^{2}\right)$ is used only for calculating $R$-factors(gt) etc. and is not relevant to the choice of reflections for refinement. $R$-factors based on $F^{2}$ are statistically about twice as large as those based on $F$, and $R$ - factors based on ALL data will be even larger.

Fractional atomic coordinates and isotropic or equivalent isotropic displacement parameters $\left(\AA^{2}\right)$

\begin{tabular}{lllll}
\hline & $x$ & $y$ & $z$ & $U_{\text {iso }} * / U_{\text {eq }}$ \\
\hline C1 & $0.67239(19)$ & $0.36402(15)$ & $0.21562(15)$ & $0.0443(5)$ \\
C2 & $0.6576(2)$ & $0.33212(18)$ & $0.31729(17)$ & $0.0556(5)$ \\
H2 & 0.7103 & 0.2710 & 0.3556 & $0.067^{*}$ \\
C3 & $0.5644(2)$ & $0.3918(2)$ & $0.3605(2)$ & $0.0694(7)$
\end{tabular}




$\begin{array}{lllll}\text { H3 } & 0.5552 & 0.3717 & 0.4294 & 0.083^{*} \\ \text { C4 } & 0.4849(3) & 0.4804(2) & 0.3038(2) & 0.0738(7) \\ \text { H4 } & 0.4200 & 0.5192 & 0.3332 & 0.089^{*} \\ \text { C5 } & 0.5005(3) & 0.51236(19) & 0.2034(2) & 0.0732(7) \\ \text { H5 } & 0.4467 & 0.5731 & 0.1652 & 0.088^{*} \\ \text { C6 } & 0.5951(2) & 0.45494(17) & 0.15921(18) & 0.0568(5) \\ \text { H6 } & 0.6070 & 0.4772 & 0.0918 & 0.068^{*} \\ \text { C7 } & 1.0474(2) & 0.29629(15) & 0.33830(14) & 0.0424(4) \\ \text { H7 } & 0.9836 & 0.2401 & 0.3570 & 0.051^{*} \\ \text { C8 } & 1.0716(2) & 0.38752(18) & 0.42469(16) & 0.0568(5) \\ \text { H8A } & 0.9739 & 0.4194 & 0.4203 & 0.068^{*} \\ \text { H8B } & 1.1338 & 0.4452 & 0.4084 & 0.068^{*} \\ \text { C9 } & 1.1495(2) & 0.3434(2) & 0.54232(16) & 0.0632(6) \\ \text { H9A } & 1.1678 & 0.4037 & 0.5958 & 0.076^{*} \\ \text { H9B } & 1.0829 & 0.2907 & 0.5610 & 0.076^{*} \\ \text { C10 } & 1.2988(2) & 0.2885(2) & 0.55192(17) & 0.0651(6) \\ \text { H10A } & 1.3416 & 0.2563 & 0.6262 & 0.078^{*} \\ \text { H10B } & 1.3701 & 0.3434 & 0.5430 & 0.078^{*} \\ \text { C11 } & 1.2786(3) & 0.1995(2) & 0.46425(18) & 0.0679(7) \\ \text { H11A } & 1.2203 & 0.1392 & 0.4805 & 0.082^{*} \\ \text { H11B } & 1.3780 & 0.1710 & 0.4682 & 0.082^{*} \\ \text { C12 } & 1.1976(2) & 0.24159(18) & 0.34615(16) & 0.0546(5) \\ \text { H12A } & 1.1787 & 0.1805 & 0.2936 & 0.065^{*} \\ \text { H12B } & 1.2625 & 0.2943 & 0.3255 & 0.065^{*} \\ \text { C13 } & 1.0145(3) & 0.4353(2) & 0.18252(19) & 0.0719(7) \\ \text { H13A } & 0.9857 & 0.4965 & 0.2197 & 0.108^{*} \\ \text { H13B } & 0.9659 & 0.4421 & 0.1027 & 0.108^{*} \\ \text { H13C } & 1.1233 & 0.4350 & 0.1996 & 0.108^{*} \\ \text { N1 } & 0.96659(17) & 0.33232(13) & 0.22141(13) & 0.0485(4) \\ \text { O1 } & 0.78750(17) & 0.17730(12) & 0.18902(14) & 0.0710(5) \\ \text { O2 } & 0.75416(18) & 0.32058(15) & 0.04381(11) & 0.0769(5) \\ \text { S1 } & 0.79429(6) & 0.29004(4) & 0.15969(4) & 0.0523(2) \\ & & & & \end{array}$

Atomic displacement parameters $\left(\AA^{2}\right)$

\begin{tabular}{lllllll}
\hline & $U^{11}$ & $U^{22}$ & $U^{33}$ & $U^{12}$ & $U^{13}$ & $U^{23}$ \\
\hline C1 & $0.0369(9)$ & $0.0421(11)$ & $0.0442(10)$ & $-0.0061(8)$ & $0.0002(7)$ & $-0.0009(8)$ \\
C2 & $0.0458(11)$ & $0.0601(14)$ & $0.0563(12)$ & $-0.0031(10)$ & $0.0104(9)$ & $0.0119(10)$ \\
C3 & $0.0505(12)$ & $0.0910(19)$ & $0.0690(15)$ & $-0.0087(13)$ & $0.0227(11)$ & $-0.0028(13)$ \\
C4 & $0.0447(12)$ & $0.0776(18)$ & $0.098(2)$ & $-0.0046(12)$ & $0.0213(13)$ & $-0.0252(15)$ \\
C5 & $0.0569(14)$ & $0.0515(14)$ & $0.098(2)$ & $0.0069(11)$ & $0.0078(13)$ & $0.0027(13)$ \\
C6 & $0.0513(12)$ & $0.0506(13)$ & $0.0579(12)$ & $0.0002(10)$ & $0.0033(10)$ & $0.0078(10)$ \\
C7 & $0.0397(10)$ & $0.0442(11)$ & $0.0410(10)$ & $-0.0014(8)$ & $0.0101(8)$ & $0.0003(8)$ \\
C8 & $0.0558(12)$ & $0.0591(13)$ & $0.0535(12)$ & $0.0128(10)$ & $0.0151(9)$ & $-0.0100(10)$ \\
C9 & $0.0655(14)$ & $0.0758(16)$ & $0.0468(12)$ & $0.0075(11)$ & $0.0163(10)$ & $-0.0124(10)$ \\
C10 & $0.0573(13)$ & $0.0805(17)$ & $0.0477(12)$ & $0.0090(11)$ & $0.0039(10)$ & $-0.0097(11)$ \\
C11 & $0.0618(14)$ & $0.0700(16)$ & $0.0583(13)$ & $0.0212(11)$ & $0.0009(10)$ & $-0.0093(11)$ \\
C12 & $0.0519(12)$ & $0.0567(13)$ & $0.0507(12)$ & $0.0091(9)$ & $0.0108(9)$ & $-0.0131(9)$
\end{tabular}




\begin{tabular}{lllllll}
$\mathrm{C} 13$ & $0.0738(15)$ & $0.0693(16)$ & $0.0675(15)$ & $-0.0071(12)$ & $0.0162(12)$ & $0.0176(12)$ \\
$\mathrm{N} 1$ & $0.0454(9)$ & $0.0507(10)$ & $0.0462(9)$ & $-0.0010(7)$ & $0.0108(7)$ & $0.0024(7)$ \\
$\mathrm{O} 1$ & $0.0658(10)$ & $0.0425(9)$ & $0.0905(11)$ & $-0.0052(7)$ & $0.0064(8)$ & $-0.0131(8)$ \\
$\mathrm{O} 2$ & $0.0797(11)$ & $0.1027(13)$ & $0.0379(8)$ & $0.0063(9)$ & $0.0050(7)$ & $-0.0119(8)$ \\
$\mathrm{S} 1$ & $0.0513(3)$ & $0.0512(3)$ & $0.0449(3)$ & $-0.0004(2)$ & $0.0027(2)$ & $-0.0090(2)$ \\
\hline
\end{tabular}

Geometric parameters $\left(\hat{A},{ }^{\circ}\right)$

\begin{tabular}{|c|c|c|c|}
\hline $\mathrm{C} 1-\mathrm{C} 6$ & $1.378(3)$ & $\mathrm{C} 9-\mathrm{C} 10$ & $1.505(3)$ \\
\hline $\mathrm{C} 1-\mathrm{C} 2$ & $1.385(3)$ & C9-H9A & 0.9700 \\
\hline $\mathrm{C} 1-\mathrm{S} 1$ & $1.760(2)$ & C9-H9B & 0.9700 \\
\hline $\mathrm{C} 2-\mathrm{C} 3$ & $1.369(3)$ & $\mathrm{C} 10-\mathrm{C} 11$ & $1.509(3)$ \\
\hline $\mathrm{C} 2-\mathrm{H} 2$ & 0.9300 & $\mathrm{C} 10-\mathrm{H} 10 \mathrm{~A}$ & 0.9700 \\
\hline $\mathrm{C} 3-\mathrm{C} 4$ & $1.364(3)$ & $\mathrm{C} 10-\mathrm{H} 10 \mathrm{~B}$ & 0.9700 \\
\hline $\mathrm{C} 3-\mathrm{H} 3$ & 0.9300 & $\mathrm{C} 11-\mathrm{C} 12$ & $1.517(3)$ \\
\hline $\mathrm{C} 4-\mathrm{C} 5$ & $1.374(4)$ & C11-H11A & 0.9700 \\
\hline $\mathrm{C} 4-\mathrm{H} 4$ & 0.9300 & C11-H11B & 0.9700 \\
\hline $\mathrm{C} 5-\mathrm{C} 6$ & $1.372(3)$ & $\mathrm{C} 12-\mathrm{H} 12 \mathrm{~A}$ & 0.9700 \\
\hline $\mathrm{C} 5-\mathrm{H} 5$ & 0.9300 & $\mathrm{C} 12-\mathrm{H} 12 \mathrm{~B}$ & 0.9700 \\
\hline $\mathrm{C} 6-\mathrm{H} 6$ & 0.9300 & $\mathrm{C} 13-\mathrm{N} 1$ & $1.462(3)$ \\
\hline $\mathrm{C} 7-\mathrm{N} 1$ & $1.481(2)$ & $\mathrm{C} 13-\mathrm{H} 13 \mathrm{~A}$ & 0.9600 \\
\hline $\mathrm{C} 7-\mathrm{C} 8$ & $1.515(3)$ & C13-H13B & 0.9600 \\
\hline $\mathrm{C} 7-\mathrm{C} 12$ & $1.516(3)$ & $\mathrm{C} 13-\mathrm{H} 13 \mathrm{C}$ & 0.9600 \\
\hline $\mathrm{C} 7-\mathrm{H} 7$ & 0.9800 & $\mathrm{~N} 1-\mathrm{S} 1$ & $1.6144(16)$ \\
\hline $\mathrm{C} 8-\mathrm{C} 9$ & $1.516(3)$ & $\mathrm{O} 1-\mathrm{S} 1$ & $1.4218(16)$ \\
\hline $\mathrm{C} 8-\mathrm{H} 8 \mathrm{~A}$ & 0.9700 & $\mathrm{O} 2-\mathrm{S} 1$ & $1.4302(15)$ \\
\hline $\mathrm{C} 8-\mathrm{H} 8 \mathrm{~B}$ & 0.9700 & & \\
\hline $\mathrm{C} 6-\mathrm{C} 1-\mathrm{C} 2$ & $120.5(2)$ & $\mathrm{H} 9 \mathrm{~A}-\mathrm{C} 9-\mathrm{H} 9 \mathrm{~B}$ & 108.0 \\
\hline $\mathrm{C} 6-\mathrm{C} 1-\mathrm{S} 1$ & $119.68(16)$ & $\mathrm{C} 9-\mathrm{C} 10-\mathrm{C} 11$ & $111.51(18)$ \\
\hline $\mathrm{C} 2-\mathrm{C} 1-\mathrm{S} 1$ & $119.84(15)$ & $\mathrm{C} 9-\mathrm{C} 10-\mathrm{H} 10 \mathrm{~A}$ & 109.3 \\
\hline $\mathrm{C} 3-\mathrm{C} 2-\mathrm{C} 1$ & $119.1(2)$ & $\mathrm{C} 11-\mathrm{C} 10-\mathrm{H} 10 \mathrm{~A}$ & 109.3 \\
\hline $\mathrm{C} 3-\mathrm{C} 2-\mathrm{H} 2$ & 120.5 & $\mathrm{C} 9-\mathrm{C} 10-\mathrm{H} 10 \mathrm{~B}$ & 109.3 \\
\hline $\mathrm{C} 1-\mathrm{C} 2-\mathrm{H} 2$ & 120.5 & $\mathrm{C} 11-\mathrm{C} 10-\mathrm{H} 10 \mathrm{~B}$ & 109.3 \\
\hline $\mathrm{C} 4-\mathrm{C} 3-\mathrm{C} 2$ & $120.8(2)$ & $\mathrm{H} 10 \mathrm{~A}-\mathrm{C} 10-\mathrm{H} 10 \mathrm{~B}$ & 108.0 \\
\hline $\mathrm{C} 4-\mathrm{C} 3-\mathrm{H} 3$ & 119.6 & $\mathrm{C} 10-\mathrm{C} 11-\mathrm{C} 12$ & $112.27(18)$ \\
\hline $\mathrm{C} 2-\mathrm{C} 3-\mathrm{H} 3$ & 119.6 & $\mathrm{C} 10-\mathrm{C} 11-\mathrm{H} 11 \mathrm{~A}$ & 109.2 \\
\hline $\mathrm{C} 3-\mathrm{C} 4-\mathrm{C} 5$ & $120.1(2)$ & $\mathrm{C} 12-\mathrm{C} 11-\mathrm{H} 11 \mathrm{~A}$ & 109.1 \\
\hline $\mathrm{C} 3-\mathrm{C} 4-\mathrm{H} 4$ & 120.0 & $\mathrm{C} 10-\mathrm{C} 11-\mathrm{H} 11 \mathrm{~B}$ & 109.1 \\
\hline $\mathrm{C} 5-\mathrm{C} 4-\mathrm{H} 4$ & 120.0 & $\mathrm{C} 12-\mathrm{C} 11-\mathrm{H} 11 \mathrm{~B}$ & 109.1 \\
\hline $\mathrm{C} 6-\mathrm{C} 5-\mathrm{C} 4$ & $120.2(2)$ & $\mathrm{H} 11 \mathrm{~A}-\mathrm{C} 11-\mathrm{H} 11 \mathrm{~B}$ & 107.9 \\
\hline $\mathrm{C} 6-\mathrm{C} 5-\mathrm{H} 5$ & 119.9 & $\mathrm{C} 11-\mathrm{C} 12-\mathrm{C} 7$ & $111.14(17)$ \\
\hline $\mathrm{C} 4-\mathrm{C} 5-\mathrm{H} 5$ & 119.9 & $\mathrm{C} 11-\mathrm{C} 12-\mathrm{H} 12 \mathrm{~A}$ & 109.4 \\
\hline $\mathrm{C} 5-\mathrm{C} 6-\mathrm{C} 1$ & $119.4(2)$ & $\mathrm{C} 7-\mathrm{C} 12-\mathrm{H} 12 \mathrm{~A}$ & 109.4 \\
\hline $\mathrm{C} 5-\mathrm{C} 6-\mathrm{H} 6$ & 120.3 & $\mathrm{C} 11-\mathrm{C} 12-\mathrm{H} 12 \mathrm{~B}$ & 109.4 \\
\hline $\mathrm{C} 1-\mathrm{C} 6-\mathrm{H} 6$ & 120.3 & $\mathrm{C} 7-\mathrm{C} 12-\mathrm{H} 12 \mathrm{~B}$ & 109.4 \\
\hline $\mathrm{N} 1-\mathrm{C} 7-\mathrm{C} 8$ & $113.90(15)$ & $\mathrm{H} 12 \mathrm{~A}-\mathrm{C} 12-\mathrm{H} 12 \mathrm{~B}$ & 108.0 \\
\hline $\mathrm{N} 1-\mathrm{C} 7-\mathrm{C} 12$ & $110.43(15)$ & $\mathrm{N} 1-\mathrm{C} 13-\mathrm{H} 13 \mathrm{~A}$ & 109.5 \\
\hline
\end{tabular}




$\begin{array}{ll}\mathrm{C} 8-\mathrm{C} 7-\mathrm{C} 12 & 110.80(15) \\ \mathrm{N} 1-\mathrm{C} 7-\mathrm{H} 7 & 107.1 \\ \mathrm{C} 8-\mathrm{C} 7-\mathrm{H} 7 & 107.1 \\ \mathrm{C} 12-\mathrm{C} 7-\mathrm{H} 7 & 107.1 \\ \mathrm{C} 7-\mathrm{C} 8-\mathrm{C} 9 & 110.76(17) \\ \mathrm{C} 7-\mathrm{C} 8-\mathrm{H} 8 \mathrm{~A} & 109.5 \\ \mathrm{C} 9-\mathrm{C} 8-\mathrm{H} 8 \mathrm{~A} & 109.5 \\ \mathrm{C} 7-\mathrm{C} 8-\mathrm{H} 8 \mathrm{~B} & 109.5 \\ \mathrm{C} 9-\mathrm{C} 8-\mathrm{H} 8 \mathrm{~B} & 109.5 \\ \mathrm{H} 8 \mathrm{~A}-\mathrm{C} 8-\mathrm{H} 8 \mathrm{~B} & 108.1 \\ \mathrm{C} 10-\mathrm{C} 9-\mathrm{C} 8 & 111.50(17) \\ \mathrm{C} 10-\mathrm{C} 9-\mathrm{H} 9 \mathrm{~A} & 109.3 \\ \mathrm{C} 8-\mathrm{C} 9-\mathrm{H} 9 \mathrm{~A} & 109.3 \\ \mathrm{C} 10-\mathrm{C} 9-\mathrm{H} 9 \mathrm{~B} & 109.3 \\ \mathrm{C} 8-\mathrm{C} 9-\mathrm{H} 9 \mathrm{~B} & 109.3\end{array}$

$\begin{array}{ll}\mathrm{N} 1-\mathrm{C} 13-\mathrm{H} 13 \mathrm{~B} & 109.5 \\ \mathrm{H} 13 \mathrm{~A}-\mathrm{C} 13-\mathrm{H} 13 \mathrm{~B} & 109.5 \\ \mathrm{~N} 1-\mathrm{C} 13-\mathrm{H} 13 \mathrm{C} & 109.5 \\ \mathrm{H} 13 \mathrm{~A}-\mathrm{C} 13-\mathrm{H} 13 \mathrm{C} & 109.5 \\ \mathrm{H} 13 \mathrm{~B}-\mathrm{C} 13-\mathrm{H} 13 \mathrm{C} & 109.5 \\ \mathrm{C} 13-\mathrm{N} 1-\mathrm{C} 7 & 118.27(15) \\ \mathrm{C} 13-\mathrm{N} 1-\mathrm{S} 1 & 118.12(13) \\ \mathrm{C} 7-\mathrm{N} 1-\mathrm{S} 1 & 118.92(12) \\ \mathrm{O} 1-\mathrm{S} 1-\mathrm{O} 2 & 119.63(10) \\ \mathrm{O} 1-\mathrm{S} 1-\mathrm{N} 1 & 107.52(9) \\ \mathrm{O} 2-\mathrm{S} 1-\mathrm{N} 1 & 107.13(10) \\ \mathrm{O} 1-\mathrm{S} 1-\mathrm{C} 1 & 107.27(10) \\ \mathrm{O} 2-\mathrm{S} 1-\mathrm{C} 1 & 106.79(9) \\ \mathrm{N} 1-\mathrm{S} 1-\mathrm{C} 1 & 108.06(8)\end{array}$

Hydrogen-bond geometry $\left(A,{ }^{\circ}\right)$

\begin{tabular}{lllll}
\hline$D-\mathrm{H} \cdots A$ & $D-\mathrm{H}$ & $\mathrm{H} \cdots A$ & $D \cdots A$ & $D-\mathrm{H} \cdots A$ \\
\hline $\mathrm{C} 2-\mathrm{H} 2 \cdots \mathrm{O} 2^{\mathrm{i}}$ & 0.93 & 2.52 & $3.268(3)$ & 137 \\
\hline
\end{tabular}

Symmetry code: (i) $x,-y+1 / 2, z+1 / 2$. 